\title{
A Nonsymmetric State-Variable Decomposition for Modal Analysis ${ }^{1}$
}

\author{
B. F. Feeny and U. Farooq \\ Michigan State University \\ Department of Mechanical Engineering \\ East Lansing, MI 48824 USA
}

\begin{abstract}
A modal decomposition strategy based on state-variable ensembles is formulated. A nonsymmetric, generalized eigenvalue problem is constructed. The data-based eigenvalue problem is related to the generalized eigenvalue problem associated with free-vibration solutions of the state-variable formulation of linear multi-degree-of-freedom systems. For linear free-response data, the inverse-transpose of the eigenvector matrix converges to the state-variable modal eigenvectors, and the eigenvalues of the nonsymmetric eigenvalue problem approximate those of the state-variable model. As such, the eigenvalues lead to estimates of frequencies and modal damping. The interpretation holds for linear systems with multi-modal free responses, whether damping is large or small, modal or nonmodal, and without the need of input data.
\end{abstract}

Keywords: state-variable modal decomposition, proper orthogonal decomposition, modal analysis, complex modes, frequency estimation, damping estimation.

\section{Introduction}

This paper presents a method of decomposing free-response ensemble data into modal components, while enabling frequency, damping, and mode-shape estimation for generally damped linear multi-degree-of-freedom systems. The basic idea is a generalization of proper orthogonal decomposition (POD) [1-3], with similarity also to smooth orthogonal decomposition (SOD) [4] and complex orthogonal decomposition (COD) [5]. Below, the application of these methods to modal analysis is reviewed, and the niche of the new method is staged.

Proper orthogonal decomposition is now a standard tool that has been applied to turbulence, structures, and many other types of systems. POD produces modes that optimize the signal energy distribution in a set of measured time series. POD has been applied, for example, to characterize spatial coherence in turbulence and structures [1-3,6-9], to evaluate the dimension of the dynamics $[3,6-8,10]$, to detect modal interactions $[11,12]$, to produce empirical modes for reduced order models [13-19], and in system identification [20-23]. The POD is similar to Karhunen-Loeve decomposition, principle components analysis, or singular value decomposition. All of these tools have been compared for structural applications [24]. The similar biorthogonal decomposition has also been applied to fluids and plasmas $[25,26]$.

POD is easily applied to discrete systems, or systems with discrete measurements $x_{i}\left(t_{j}\right)$, where $i=1, \ldots, M$ are the sensor indices, and $j=1, \ldots, N$ are the time sample indices. An ensemble matrix is formed as $\mathbf{X}^{T}=\left[\mathbf{x}_{1}, \ldots, \mathbf{x}_{M}\right]$, where the vectors $\mathbf{x}_{i}$ have elements of time samples $x_{i}\left(t_{j}\right)$. So each row of $\mathbf{X}$ is a sampled history of one sensor on the structure. Then the matrix $\mathbf{R}=\mathbf{X X}^{T} / N$ is formed. If the means of the signals are all zero, then $\mathbf{R}$ is

\footnotetext{
${ }^{1}$ Journal of Sound and Vibration 310 (4-5) 792-800 (2008).
} 
a covariance matrix. Otherwise, we will refer to it as a correlation matrix, and the method can still be applied. The eigenvectors of $\mathbf{R}$ are the proper orthogonal modes (POMs), and the eigenvalues are the proper orthogonal values.

If the mass distribution is uniform, the POD produces the normal modes of a structure [27-30], including 2-D structures [31]. If the mass distribution is not uniform, but known, or if the stiffness matrix is known, the POD can be weighted to produce the normal modes [27]. POD can be used for modal analysis if the damping is light, and if the system is either impulse excited or randomly excited [32]. The proper orthogonal modes (POMs) then converge to the linear normal modes, and the proper orthogonal values provide the mean squared values of the modal coordinates.

The related SOD can be applied in inhomogeneous, lightly damped cases to find structural modes [4]. In this case, an ensemble $\mathbf{V} \approx \dot{\mathbf{X}}$ of velocities is formed. This can be done by finite difference through a matrix $\mathbf{D}$, such that $\mathbf{V}=\mathbf{X D}^{T}$. The velocity covariance matrix $\mathbf{S}=\mathbf{V V}^{T} / N_{v}$ is formed, where $N_{v}$ is the number of velocity samples. Then the generalized eigenvalue problem is written as $\lambda \mathbf{R} \underline{\psi}=\mathbf{S} \underline{\psi}$. For a free multi-modal response with light damping, the eigenvalues $\lambda$ approximate the frequencies squared, and the inverse-transpose of the modal matrix $\Psi$ approximates the linear modal matrix. (Cast this way, the method might aptly be called "frequency decomposition.")

Another decomposition technique is the complex orthogonal decomposition (COD) [5]. Here, the ensemble $\mathbf{X}$ of oscillatory signals is expanded into the complex domain to form an ensemble $\mathbf{Z}$ of complex analytic signals. This is done non-uniquely by the FFT [33] or Hilbert transform [33,34]. Then a complex Hermitian correlation matrix $\mathbf{R}=\mathbf{Z} \overline{\mathbf{Z}}^{T} / N$ is formed. (A complex Hermitian matrix was also formed in the frequency domain for modal analysis [35].) The complex Hermitian matrix $\mathbf{R}$ has real eigenvalues and generally complex eigenvectors (COMs). The eigenvalues represent mean squared amplitudes of complex modal oscillations. The COMs can be interpreted as complex modes, representing generally a mix of traveling and standing wave behaviors, which can be extracted and quantified. As such, the COD can characterize the modal content of systems with mixed standing and traveling wave behavior. Initial indications are that the complex modes can be efficiently extracted. Much like POD for general systems, the COMs will not produce complex linear normal modes except under special circumstances. But our ongoing work suggests that the COD provides an optimized and systematic characterization of generally oscillatory behavior.

The approach presented here is a generalized eigen decomposition based on state-variable ensembles. We will tie this decomposition strategy to state-variable models of generally damped linear vibration systems to reveal a method of obtaining frequency, damping, and mode shape information, without access to input measurements. The method will be illustrated with numerical examples.

\section{Nonsymmetric state-variable modal decomposition}

In this section, we summarize the state-variable modeling of vibration systems. The decomposition equation is presented, and then tied to the state-variable model. 


\subsection{State-variable linear vibration model}

The state-variable model of linear vibration systems is used on systems with nonproportional (non-Rayleigh [36]) and nonmodal (non-Caughey [37]) damping to obtain damped vibration modes $[38,39]$. The equations of motion for free vibrations are

$$
\mathbf{M} \ddot{\mathbf{x}}+\mathbf{C} \dot{\mathbf{x}}+\mathbf{K x}=\mathbf{0},
$$

where $\mathbf{x}$ is an $n \times 1$ array of mass displacements, $\mathbf{M}, \mathbf{C}$, and $\mathbf{K}$, are the $n \times n$ mass, damping, and stiffness matrices, and the dots indicate time derivatives. Then defining a $2 n \times 1$ state vector $\mathbf{y}^{T}=\left[\dot{\mathbf{x}}^{T}, \mathbf{x}^{T}\right]$, and introducing the equation $\mathbf{M} \dot{\mathbf{x}}-\mathbf{M} \dot{\mathbf{x}}=\mathbf{0}$, yields unforced equations of motion of the form

$$
\mathrm{A} \dot{\mathbf{y}}+\mathrm{By}=\mathbf{0},
$$

where

$$
\mathbf{A}=\left[\begin{array}{cc}
\mathbf{0} & \mathbf{M} \\
\mathbf{M} & \mathbf{C}
\end{array}\right], \quad \mathbf{B}=\left[\begin{array}{cc}
-\mathbf{M} & \mathbf{0} \\
\mathbf{0} & \mathbf{K}
\end{array}\right]
$$

$\mathbf{A}$ and $\mathbf{B}$ are $2 n \times 2 n$ and symmetric, but are neither positive nor negative definite.

Assuming a response of the form $\mathbf{y}=e^{\alpha t} \underline{\phi}$, the eigenvalue problem

$$
\alpha \mathbf{A} \underline{\phi}+\mathbf{B} \underline{\phi}=\mathbf{0}
$$

in general yields complex eigenvalues $\alpha$ and eigenvectors $\phi$, with $\phi=\left[\mathbf{v}^{T}, \mathbf{w}^{T}\right]^{T}$, where $n \times 1$ vector partition $\mathbf{v}$ corresponds to characteristic shapes of velocity states, and partition $\mathbf{w}$ represents characteristic shapes in displacement (complex modes). By the construction of $\mathbf{y}$, $\mathbf{v}=\alpha \mathbf{w}$. The vectors $\phi$ are orthogonal with respect to matrices $\mathbf{A}$ and $\mathbf{B}$. The latter does not imply that the vectors $\mathbf{w}$ are orthogonal with respect to $\mathbf{M}$ and $\mathbf{K}$. In fact, they are not.

\subsection{Decomposition strategy}

The decomposition strategy is based on the free-response state-variable ensemble $\mathbf{Y}=$ $\left[\mathbf{V}^{T}, \mathbf{X}^{T}\right]^{T}$, where $\mathbf{X}$ is a displacement ensemble, and $\mathbf{V}=\mathbf{X D}^{T} \approx \dot{\mathbf{X}}$ is an approximate velocity ensemble, like the SOD [4]. An example of an $\left(N-2 n_{d}\right) \times N$ matrix of centered finite differences, with $n_{d}=1$ for half the span of the finite difference, is

$$
\mathbf{D}=\frac{1}{2 \Delta t}\left[\begin{array}{cccccc}
-1 & 0 & 1 & 0 & \ldots & 0 \\
0 & -1 & 0 & 1 & \ldots & 0 \\
\vdots & \ddots & \ddots & \ddots & \ddots & \vdots \\
0 & \ldots & \ldots & -1 & 0 & 1
\end{array}\right]
$$

where $\Delta t$ represents the sampling time. Thus $\mathbf{V}$ is $n \times\left(N-2 n_{d}\right)$, and so the first and last $n_{d}$ columns of $\mathbf{X}$ are dropped so that $\mathbf{Y}$ has compatible partitions.

We then take the derivative $\mathbf{W}=\mathbf{Y D}^{T} \approx \dot{\mathbf{Y}}$, this time using an $\left(N-4 n_{d}\right) \times\left(N-2 n_{d}\right)$ difference matrix $\mathbf{D}$. The first and last $n_{d}$ time samples of $\mathbf{Y}$ are then dropped so that the dimensions of $\mathbf{Y}$ and $\mathbf{W}$ are both $2 n \times N-4 n_{d}$. We form a correlation matrix $\mathbf{R}=$ $\mathbf{Y} \mathbf{Y}^{T} /\left(N-4 n_{d}\right)$ and a nonsymmetric matrix $\mathbf{N}=\mathbf{Y} \mathbf{W}^{T} /\left(N-4 n_{d}\right)$. 
The eigenvalue problem is then

$$
\alpha \mathbf{R} \underline{\psi}=\mathbf{N} \underline{\psi} .
$$

Rewriting this eigenvalue problem, $\alpha \mathbf{Y} \mathbf{Y}^{T} \underline{\psi}=\mathbf{Y W}^{T} \underline{\psi}$. Making use of Eq. (2), $\mathbf{W} \approx \dot{\mathbf{Y}}=$ $-\mathbf{A}^{-1} \mathbf{B Y}$, and we have

$$
\alpha \mathbf{Y} \mathbf{Y}^{T} \underline{\psi} \approx-\mathbf{Y Y}^{T} \mathbf{B}^{T} \mathbf{A}^{-T} \underline{\psi}
$$

We expect $\mathbf{Y} \mathbf{Y}^{T}$ to be invertible if all displacement measurements are independent and if $N-4 n_{d}>n$.

As such, $\alpha \underline{\psi} \approx-\mathbf{B}^{T} \mathbf{A}^{-T} \underline{\psi}$. In matrix form

$$
\boldsymbol{\Psi} \Lambda \approx-\mathbf{B}^{T} \mathbf{A}^{-T} \boldsymbol{\Psi}
$$

where $\boldsymbol{\Lambda}$ is a diagonal matrix of eigenvalues. Taking the inverse-transpose yields $\boldsymbol{\Psi}^{-T} \boldsymbol{\Lambda}^{-1} \approx$ $-\mathbf{B}^{-1} \mathbf{A} \boldsymbol{\Psi}^{-T}$, whence $\mathbf{B} \boldsymbol{\Psi}^{-T} \approx-\mathbf{A} \boldsymbol{\Psi}^{-T} \boldsymbol{\Lambda}$, and hence

$$
-\mathbf{A}^{-1} \mathbf{B} \Psi^{-T} \approx \Psi^{-T} \Lambda
$$

Letting $\mathbf{U}=\mathbf{\Psi}^{-T}$, the data eigenvalue problem leads to

$$
-\mathbf{A}^{-1} \mathbf{B U} \approx \mathbf{U} \Lambda
$$

which is a generalized eigenvalue problem with matrices $\mathbf{A}$ and $\mathbf{B}$, the solution of which determines the unknowns $\mathbf{U}$ and $\boldsymbol{\Lambda}$. The matrix form of the structural eigenvalue problem of Eq. (4) is

$$
-\mathbf{A}^{-1} \mathbf{B} \Phi=\Phi \Gamma
$$

a generalized eigenvalue problem with the same matrices $\mathbf{A}$ and $\mathbf{B}$, the solution of which determines the unknowns $\boldsymbol{\Phi}$ and $\boldsymbol{\Gamma}$. The eigenvalue problems of Eqs. (10) and (11) have the same solution (within the modal normalization constants), indicating that $\boldsymbol{\Phi} \approx \mathbf{U}=\mathbf{\Psi}^{-T}$ and $\boldsymbol{\Gamma}=\boldsymbol{\Lambda}$. (The same logic is seen in SOD analysis of linear vibration systems [4].)

Thus, we expect the eigenvalues of Eq. (6) to approximate the state-variable eigenvalues, containing information about damping and frequency. The inverse of the modal matrix from Eq. (6) resembles the complex linear normal modal matrix of the state-variable system Eq. (4), and contains velocity and displacement partitions. The only approximation in the method is in $\dot{\mathbf{X}} \approx \mathbf{X D}^{T}$ and $\dot{\mathbf{Y}} \approx \mathbf{Y D}^{T}$. Hence we expect reasonable estimations when noise is limited and the step size is sufficiently small compared to characteristic time scales.

\section{Numerical examples}

For comparison, we use the example shown in [27] for POD and [4] for SOD. We will present underdamped and overdamped examples for both proportional and generalized damping. The mass and stiffness matrices are

$$
\mathbf{M}=\left[\begin{array}{lll}
2 & 0 & 0 \\
0 & 1 & 0 \\
0 & 0 & 1
\end{array}\right], \mathbf{K}=\left[\begin{array}{rrr}
2 & -1 & 0 \\
-1 & 2 & -1 \\
0 & -1 & 1
\end{array}\right]
$$


and the initial conditions were $\mathbf{x}(0)=(1,0,0)^{T}$, and $\mathbf{v}(0)=(0,0,0)^{T}$. The undamped natural frequencies of the system are $\omega_{1}=0.4209, \omega_{2}=1.000$, and $\omega_{3}=1.6801$.

In each simulation, the time responses for each mass displacement and velocity were numerically computed. Here, we used a step size $\Delta t=0.0049767$ and a sample size $N=3000$. This time record closely matches one period of the lowest-frequency mode. We generated the state variable response from $\mathbf{y}(t)=\mathbf{\Phi} \mathbf{q}_{0} e^{\alpha t}$ where $\mathbf{q}_{0}=\Phi^{T} \mathbf{A y}(0)$. To emulate an experiment, we kept the displacement ensemble $\mathbf{X}$ from the displacement partition of the ensemble generated from sampling $\mathbf{y}(t)$. We approximated $\dot{\mathbf{X}} \approx \mathbf{V}=\mathbf{X D}^{T}$, and built the state-variable data ensemble $\mathbf{Y}$. The ensemble $\mathbf{W}=\mathbf{Y D}^{T}$ (independent from displacement modal vector $\mathbf{u}$ ) was then formed and the endpoints were truncated to obtain compatible matrix dimensions. The eigenvalue problem Eq. (6) was formulated using $\mathbf{Y}$ and $\mathbf{W}$.

\subsection{Proportionally damped systems}

In this example we chose the damping matrix to be proportional to just the mass matrix, such that $\mathbf{C}=c \mathbf{M}$.

First, we consider an underdamped example with $c=0.5$. The system eigenvalues are complex, of the form $-\zeta_{j} \omega_{j} \pm i \omega_{j} \sqrt{1-\zeta_{j}^{2}}$. These values are $\alpha_{1}=\bar{\alpha}_{1}=0.25+0.3386 i$, $\alpha_{3}=\bar{\alpha}_{4}=0.25+0.9682 i, \alpha_{5}=\bar{\alpha}_{6}=0.25+1.6614 i$. The modal damping factors are then $\zeta_{1}=0.5940, \zeta_{2}=0.2500$ and $\zeta_{3}=0.1488$. The lowest-frequency mode has a damped frequency of $\omega_{d 1}=0.3386$. Our time record specified above spans $81 \%$ of the associated oscillation period.

From the decomposition eigenvalues, estimates of the damping factors and natural frequencies were identical to that of system to more than four significant figures. For proportional damping three out of six of the displacement partitions of the state-variable modal vectors are independent. The corresponding independent displacement eigenvectors were normalized to real form of unit length as $\mathbf{u}_{1}^{T}=(0.3602,0.5928,0.7204)^{T}, \mathbf{u}_{2}^{T}=$ $(-0.7071,0.0,0.7071)^{T}$ and $\mathbf{u}_{3}^{T}=(0.2338,-0.8524,0.4676)^{T}$. All agree with the linear normal modes (LNMs) to 4th decimal place.

Now we consider an overdamped example with $c=2.5$, leading to two complex and four real structural eigenvalues. The complex eigenvalues are $\alpha_{1}=\bar{\alpha}_{1}=1.25+1.1227 i$, whereas the real pairs are $(2.427,0.073)$ and $(2.000,0.500)$, which have modal damping factors of $\zeta_{1}=2.9701, \zeta_{2}=1.2500$ and $\zeta_{3}=0.7440$. The decomposition method also yielded one complex and two real eigenvalues from which damping factors and natural frequencies are identical to those of the structural problem. Also, the corresponding independent displacement eigenvectors, normalized to real form of unit length agreed with the LNMs to the fourth decimal place.

\subsection{Generally damped systems}

We will consider underdamped and overdamped examples. For the underdamped example the damping matrix was 


$$
\mathbf{C}=\left[\begin{array}{rrr}
1.2 & -0.9 & 0 \\
-0.9 & 1.2 & -0.3 \\
0 & -0.3 & 0.3
\end{array}\right]
$$

Then $\mathbf{K M}^{-1} \mathbf{C} \neq \mathbf{C M}^{-1} \mathbf{K}$, rendering the system to be nonmodally damped [37]. In the case of nonmodal damping, the definition of a modal damping factor is ill posed. Nonetheless, the frequency of damped oscillation is the imaginary part of the eigenvalue. The eigenvalues obtained from the structural-model eigenvalue problem Eq. (4) are complex, and are $\alpha_{1}=$ $\bar{\alpha}_{2}=0.7620+1.4190 i, \alpha_{3}=\bar{\alpha}_{4}=0.2474+1.006 i, \alpha_{5}=\bar{\alpha}_{6}=0.0405+0.4218 i$. Using the magnitude of the eigenvalue as " $\omega_{j}$ ", and and comparing the real part to " $\zeta_{j} \omega_{j}$ " produces numbers for $\tilde{\zeta}_{j}$, and $\tilde{\omega}_{j}$. In this case $\tilde{\zeta}_{1}=0.0957, \tilde{\zeta}_{2}=0.2388$, and $\tilde{\zeta}_{3}=0.4731$, and $\tilde{\omega}_{1}=0.4238, \tilde{\omega}_{2}=1.0360$, and $\tilde{\omega}_{3}=1.6107$.

The modal decomposition eigenvalues were found to be nearly identical to the system structural eigenvalues, and the "damping factors" and "natural frequencies" were identical to fourth decimal place. All unit length normalized displacement partitions of the corresponding complex eigenvectors, $\mathbf{w}_{1}^{T}=\overline{\mathbf{w}}_{2}^{T}=(-0.3674+0.0317 i,-0.5901-0.0129 i,-0.7197-0.0056 i)^{T}$, $\mathbf{w}_{3}^{T}=\overline{\mathbf{w}}_{4}^{T}=(0.6517+0.1358 i, 0.0499-0.1539 i,-0.7908+0.1022 i)^{T}, \mathbf{w}_{5}^{T}=\overline{\mathbf{w}}_{6}^{T}=(-0.3526+$ $0.1499 i, 0.9224-0.0431 i,-0.3469-0.2670 i)^{T}$, agreed with the LNMs to the fourth decimal place.

Now a highly overdamped example is considered. With the damping matrix chosen as

$$
\mathbf{C}=\left[\begin{array}{rrr}
6.0 & -4.5 & 0 \\
-4.5 & 7.5 & -3.0 \\
0 & -3.0 & 3.0
\end{array}\right]
$$

the structural modal eigenvalue problem (4) produces four real and two complex eigenvalues. The complex eigenvalues are $\alpha_{1}=\bar{\alpha}_{1}=0.1858+0.4165 i$, whereas the real pairs are $(9.8853,0.2432)$ and $(2.6180,0.3820)$. The "damping factors" are $\tilde{\zeta}_{1}=0.4073, \tilde{\zeta}_{2}=1.5000$ and $\tilde{\zeta}_{3}=3.2665$ and corresponding "modal frequencies" are $\tilde{\omega}_{1}=0.4561, \tilde{\omega}_{2}=1.000$, and $\tilde{\omega}_{3}=1.5504$.

The decomposition method produced four real and two complex eigenvalues nearly identical to the structural eigenvalues. The displacement partitions of the six eigenvectors were $\mathbf{w}_{1}^{T}=\overline{\mathbf{w}}_{2}^{T}=(0.4717-0.0829 i, 0.5784+0.0054 i, 0.6728+0.0534 i)^{T}, \mathbf{w}_{3}^{T}=(0.5365,0,-0.8439)^{T}$, $\mathbf{w}_{4}^{T}=(0.1989,0,-0.9800)^{T}, \mathbf{w}_{5}^{T}=(-0.2792,0.8869,-0.3680)^{T}$, and $\mathbf{w}_{6}^{T}=(0.1098,-0.7683$, $-0.6305)^{T}$, which agreed with the LNMs to the 4 th decimal place.

The differentiation time step is a parameter of the method. If the step size is too large, it results in erroneous parameter estimates. This implies that the sampling resolution needs to be chosen appropriately to fully capture the features of the transient time response, and in turn to accurately estimate the system parameters, particularly for very highly damped (or very fast) systems. For choosing the sample time, important features of the response are the characteristic settling time, $\tau_{1 j}=4 / \zeta_{j} \omega_{j}$, and the oscillation period, $\tau_{2 j}=2 \pi / \omega_{d j}$. Our experience with numerous simulations suggests that any step size that lies within $5 \%$ of the minimum (over $j$ ) of $\tau_{1 j}$ and $\tau_{2 j}$ would give reasonable resolution. For data obtained through experiments, the settling time criterion might be good enough to approximate the step size. 
Time records should be appropriately chosen with regard to the maximum of of $\tau_{1 j}$ and $\tau_{2 j}$. This information may not be available a-priori, but provides a rough rule of thumb for the required time resolution.

\section{Robustness under noise}

To emulate a 10-bit quantization noise in digitization, we set up random fluctuations. The MATLAB function "rand" was used to generate an ensemble of uniformly distributed random numbers between $\pm 2^{-10} x_{0}$, where $x_{0}$ is the maximum initial condition. 'Rand' emulates white noise. We verified that the spectrum of our random data was uniformly distributed. The root mean squared value was very close to $2^{-10} x_{0} / \sqrt{3}$. The random ensemble was added to the displacement matrix $\mathbf{X}$. We then took numerical derivatives of the new $\mathbf{X}$ to build $\mathbf{V}$ and subsequently our ensemble matrices. All the above mentioned cases were studied under noisy data with a step size of 0.0049767 and $N=3000$. To reduce noise amplification from differentiation over short time steps, we used a differentiation step size of $32\left(n_{d}=16\right)$. Examples of finite-difference mass accelerations with noise are shown in Figure 1 for the proportionally underdamped and overdamped examples. The figure also shows how much time record was used in all previous examples.

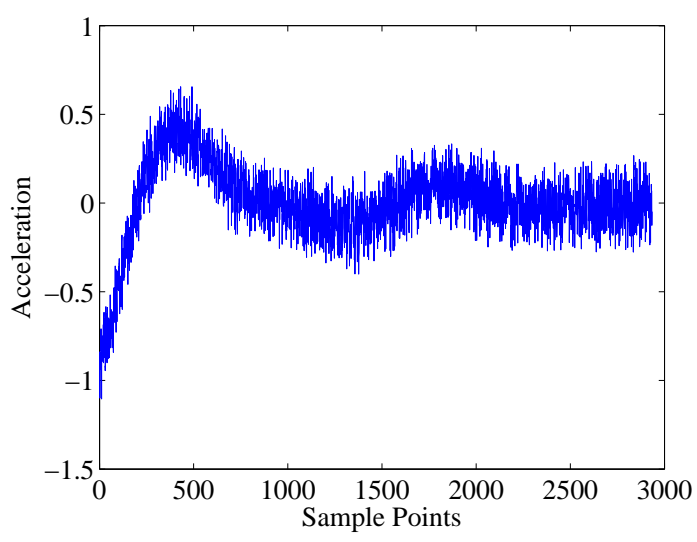

(a)

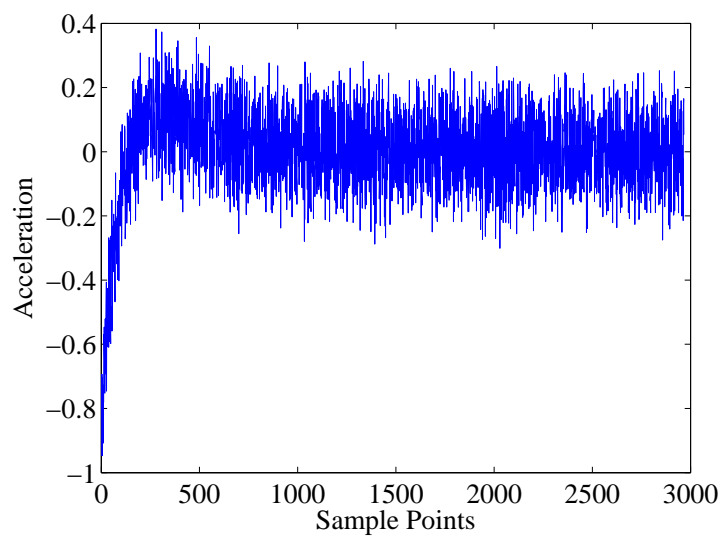

(b)

Figure 1: The acceleration of mass one, computed from applying the finite difference matrix $\mathbf{D}$ to the displacement ensemble with noise. (a) is the underdamped example, and (b) is the overdamped example.

For the proportionally damped, underdamped example of $c=0.5$, the errors in the estimated damping factors and natural frequencies were $0.82 \%, 0.57 \%$ and $0.17 \%$, and $0.09 \%$, $0.05 \%$ and $0.01 \%$, respectively. The corresponding complex independent displacement eigenvectors normalized to real form agreed with the LNMs to the 4th decimal place. For the overdamped case of $c=2.5$, we were able to get good estimates of slow eigenvalues. The slow eigenvalues were 0.0743 and 0.4095 , with corresponding vectors $(0.3329,0.5884,0.7369)^{T}$ and $(0.6968,0.0221,-0.7169)^{T}$ that are comparable to the state-variable model eigenvalues $(0.073$ and 0.500$)$ and vectors $(0.3602,0.5928,0.7204)^{T}$ and $(0.7071,0.0,-0.7071)^{T}$ respec- 


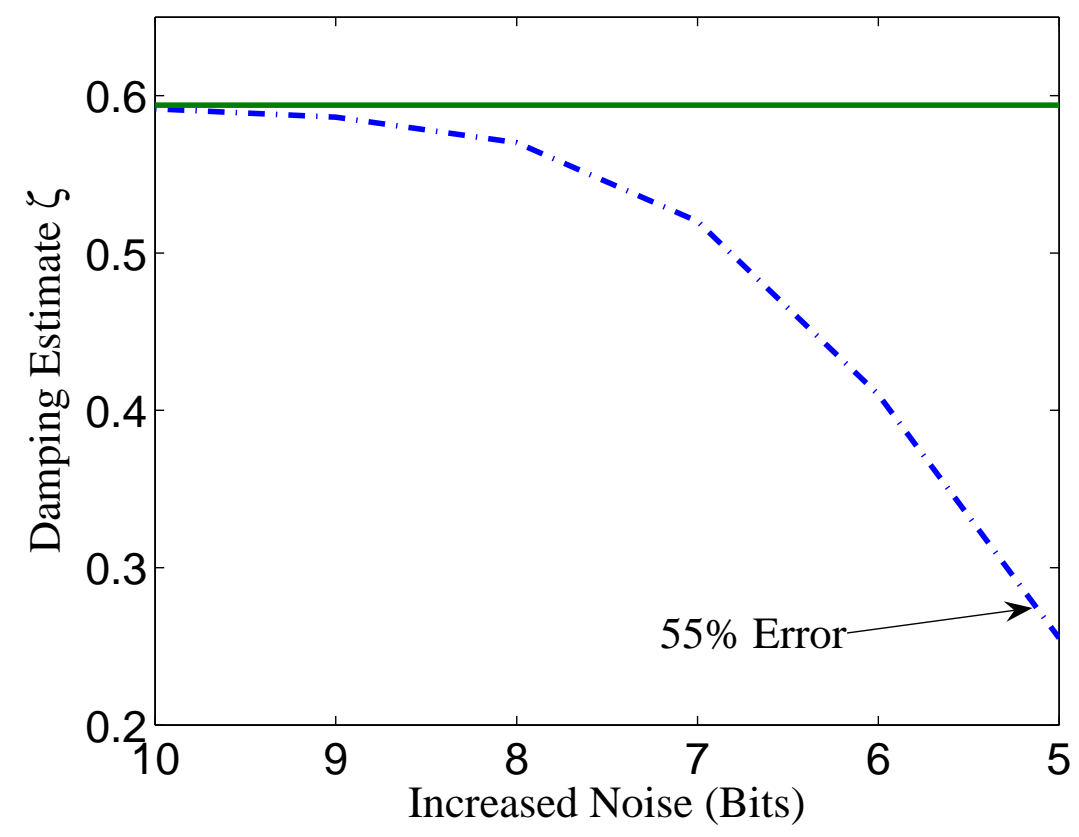

Figure 2: The damping factor estimate for first mode for various levels of noise. The solid line (-) shows system damping while dashed line (- -) presents the estimated damping. The horizontal axis indicates the value $b$ representing the uniform noise in the range $\pm 1 / 2^{b}$.

tively. But the fast eigenvalues, even with faster sampling and a larger differentiation step size, were corrupted by noise and gave erroneous results. This means we were unable to get good estimates of $\zeta_{j}$ and $\omega_{j}$ for the overdamped models. From the slow eigenvalues though, we can estimate modal time constants and given the system frequencies (e.g. by FEM), we can still estimate damping ratios with reasonable accuracy.

In generally damped examples, for the underdamped case of Eq. (13), the errors in the estimated damping factors and natural frequencies were $0.52 \%, 0.04 \%$ and $0.67 \%$, and $0.04 \%$, $0.11 \%$ and $0.13 \%$, respectively. The displacement partitions of the six complex eigenvectors agreed with the LNMs to the 3rd decimal place. For overdamped case of Eq. (14), the same interpretation holds as described in case of proportional damped overdamped case.

Lastly, we considered the effect of increasing the noise level. Here the system was chosen to be modally under damped with $\mathbf{C}=0.5 \mathbf{M}$. We used a differentiation step size of 32 $\left(n_{d}=16\right)$. As the noise bit level was incremented (doubling the noise level), the error typically increased, until at five bits, the error in the worst mode (highest damping factor) was $55 \%$ (Figure 2 and Figure 3). That some frequency estimates improved with noise is probably random happenstance.

We briefly looked into varying the number of data. The results were similar with $N=$ 3730 (a full response cycle of the lower mode damped response), and the results deteriorated, primarily for the lowest mode, when $N$ was increased to 9000 . The suspected reason is that, with the modal damping factor of 0.5940 , the modal response was settled for most of the time record, and therefore the lowest modal data was dominated by the noise. In fact, one 


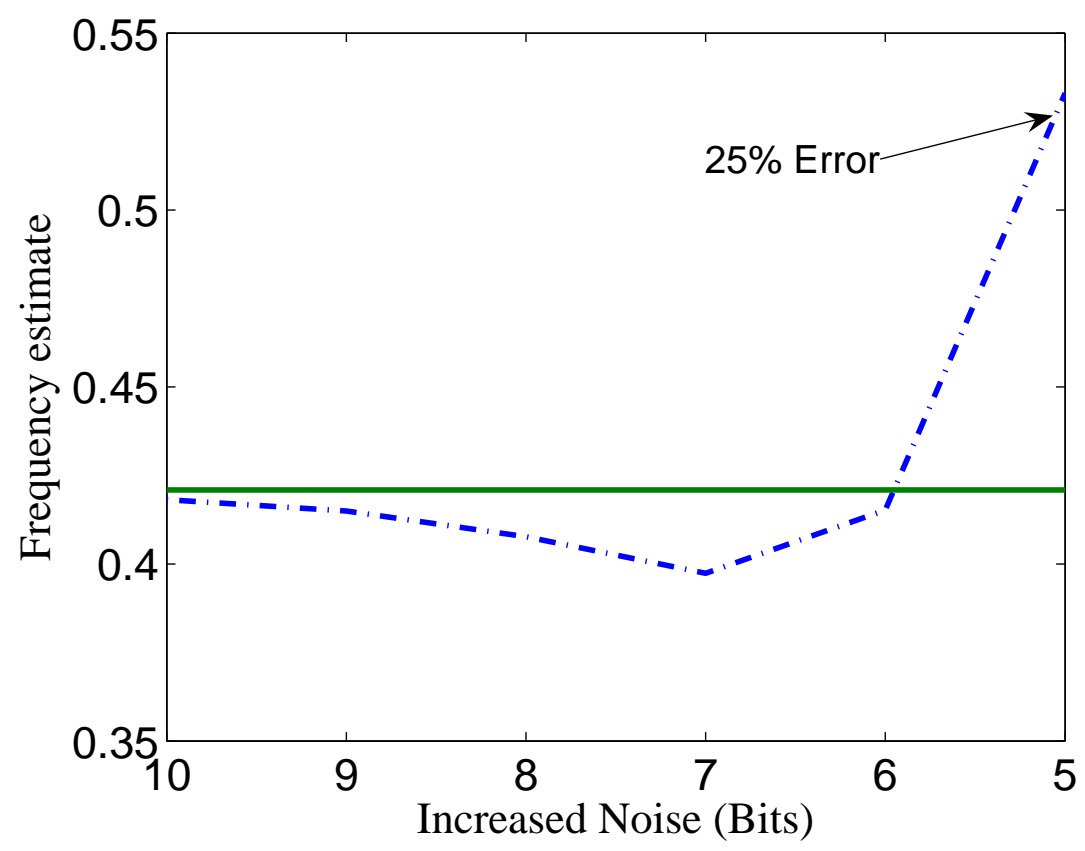

Figure 3: The modal frequency estimate for first mode for various levels of noise. The solid line $(-)$ shows modal frequency while dashed line (- -) presents the estimated frequency. The horizontal axis indicates the value $b$ representing the uniform noise in the range $\pm 1 / 2^{b}$.

of the better results came with $N=1865$, representing a half cycle of lower mode response. For further lower values of $N$, results deteriorated. It seems that the time record should include at least a half cycle of the mode to be identified, but also for modes with significant damping, the time record should not be too long. Observations with various step sizes were also made. When noise is present, longer differentiation step sizes are beneficial at larger noise levels. However, the differentiation step size promotes error if it is a "large" fraction of the response period of the mode to be identified. These are simple observations from a few numerical experiments. A systematic study is targeted for ongoing studies.

These examples suggest that the decomposition method is robust under low levels of noise that might be seen in experiments. In multiple simulations, we found that examples with damping factors up to around $\zeta=0.8$ were successful. With higher damping we could estimate slow time constants but not $\zeta_{j}$ and $\omega_{j}$. In many experiments, accelerometers are used so displacement signals will be less prone to high frequency noise since accelerations are integrated to obtain $X$ and $V$.

\section{Conclusion}

We have proposed a method for extracting modal information from free responses of generally damped linear multi-degree-of-freedom systems. A generalized unsymmetric eigenvalue problem was posed, involving "correlations" of state-variable ensembles. The resulting 
eigenvalues and eigenvectors can be complex in general. The eigenvalues hold information of damping and frequency, while the eigenvectors indicate the mode shapes associated with the state-variable model.

The method is applicable to free multi-modal responses with small or "large" damping, and with modal or nonmodal damping. Under these conditions, the system input need not be measured; only the response is measured.

We applied this scheme to several numerical examples, for which accurate estimates of modal parameters (frequency, damping factor or decay rate, and mode shapes) were possible regardless of the damping value. The sampling rate should be sufficiently smaller than the shortest characteristic time interval, defined by decay time or oscillations period. For nonmodally damped systems, the eigenvalues can accurately quantify the decay rates, damped frequencies, and mode shapes, including complex modes. In the presence of noise, large damping factors could not be extracted, but the slow decay rates were still estimated. Numerical examples suggest that there is an optimal choice of the differentiation time step, and the time record, for extracting modal frequencies and damping in the presence of noise.

\section{Acknowledgement}

This material is based upon work supported by the National Science Foundation under Grant No. CMMI-0727838. Any opinions, findings, and conclusions or recommendations expressed in this material are those of the authors and do not necessarily reflect the views of the National Science Foundation.

\section{References}

[1] J. L. Lumley, The structure of inhomogeneous turbulent flow, in Atmospheric Turbulence and Radio Wave Propagation (A. M. Yaglom and V. I. Tatarski, eds.), (Nauka, Moscow), 1967.

[2] J. L. Lumley, Stochastic Tools in Turbulence. Academic Press, New York., 1970.

[3] G. Berkooz, P. Holmes, and J. L. Lumley, The proper orthogonal decomposition in the analysis of turbulent flows, Annual reviews of Fluid mechanics, vol. 25, pp. 539-575, 1993.

[4] D. Chelidze and W. Zhou, Smooth orthogonal decomposition-based vibration mode identification, Journal of Sound and Vibration, vol. 292, pp. 461-473, 2006.

[5] B. F. Feeny, A complex orthogonal decomposition for wave motion analysis, Journal of Sound and Vibration, in press, 2007.

[6] J. P. Cusumano and B. Y. Bai, Period-infinity periodic motions, chaos and spatial coherence in a 10 degree of freedom impact oscillator, Chaos, Solitons and Fractals, vol. 3, no. 5, pp. 515-535, 1993. 
[7] J. P. Cusumano, M. T. Sharkady, and B. W. Kimble, Spatial coherence measurements of a chaotic flexible-beam impact oscillator, in Aerospace Structures: Nonlinear Dynamics and System Response, vol. ASME AD-Vol. 33, pp. 13-22, 1993.

[8] B. I. Epureanu, L. S. Tang, and M. P. Paidoussis, Exploiting chaotic dynamics for detecting parametric variations in aeroselastic systems, AIAA Journal, vol. 42, no. 4, pp. 728-735, 2004.

[9] L. Ukeiley, M. Varghese, M. Glauser, and D. Valentine, Multifractal analysis of a lobed mixer flowfield utilizing the proper orthogonal decomposition, AIAA Journal, vol. 30, no. 5, pp. 1260-1267, 1993.

[10] L. Ukeiley, M. Glauser, and D. Wick, Downstream evolution of proper orthogonal decomposition eigenfunctions in a lobed mixer, AIAA Journal, vol. 31, no. 8, pp. 1392-1397, 1993.

[11] M. A. Davies and F. C. Moon, Solitons, chaos, and modal interactions in periodic structures, in Nonlinear Dynamics: the Richard Rand 50th Anniversary Volume, pp. 119-143, Singapore: World Scientific, 1997.

[12] I. T. Georgiou, I. B. Schwartz, E. Emaci, and A. Vakakis, Interaction between slow and fast oscillations in an infinite degree of freedom linear system coupled to a nonlinear subsystem, Journal of Applied Mechanics, vol. 66, no. (2), pp. 448-459., 1999.

[13] P. M. FitzSimons and C. Rui, Determining low dimensional models of distributed systems, Advances in Robust and Nonlinear Control Systems, vol. ASME-DSC53, pp. 9-15, 1993.

[14] G. Kerschen, J. Golinval, A. Vakakis, and L.A. Bergman, The method of proper orthogonal decomposition for dynamical characterization and order reduction of mechanical systems: an overview, Nonlinear Dynamics. Special issue: Dimension Reduction of Dynamical Systems: Methods, Models, Applications., vol. 41, pp. 141-170, 2005.

[15] R. V. Kappagantu and B. F. Feeny, Part 2: Proper orthogonal modal modeling of a frictionally excited beam, Nonlinear Dynamics, vol. 23, no. 1, pp. 1-11, 2000.

[16] R. Kappagantu and B. Feeny, An "optimal" modal reduction of a system with frictional excitation, Journal of Sound and Vibration, vol. 224, no. 5, pp. 863-877, 1999.

[17] B. I. Epureanu, E. H. Dowell, and K. C. Hall, Reduced-order models of unsteady transonic viscouls flows in turbomachinery, Journal of Fluids and Structures, vol. 14, pp. 1215-1234, 2000.

[18] B. I. Epureanu, A parametric analysis of reduced order models of viscous flows in turbomachinery, Journal of Fluids and Structures, vol. 17, pp. 971-982., 2003.

[19] G. Kerschen, B. F. Feeny, and J. C. Golinval, On the exploitation of chaos to produce reduced order models, Computer Methods in Applied Mechanics and Engineering, vol. 192, pp. 1785-1795, 2003. 
[20] K. Yasuda and K. Kamiya, Experimental identification technique of nonlinear beams in time domain, in ASME Design Engineering Technical Conferences, (Sacramento), 1997. on CD-ROM.

[21] X. Ma and A. F. Vakakis, Karhunen-loève decomposition of the transient dynamics of a multibay truss, AIAA Journal, vol. 37, no. (8), pp. 939-946., 1999.

[22] X. Ma, M. A. F. Azeez, and A. F. Vakakis, Nonlinear normal modes and nonparametric system identification of nonlinear oscillators, Mechanical Systems and Signal Processing, vol. 14, no. (1), pp. 37-48., 2000.

[23] V. Lanaerts, G. Kerschen, and J. C. Golinval, Parameter identification of nonlinear mechanical systems using proper orthogonal decomposition, in Proceedings of the IMAC XVIII, (San Antonio.), 2000.

[24] Y. C. Liang, H. P. Lee, S. P. Lim, W. Z. Lin, K. H. Lee, and C. G. Wu, Proper orthogonal decomposition and its applications - part 1: Theory, Journal of Sound and Vibration, vol. 252(3), pp. 527-544, 2002.

[25] N. Aubry, R. Guyonnet, and R. Lima, Spatiotemporal analysis of complex signals: Theory and applications, Journal of Statistical Physics, vol. 64, no. (3-5), pp. 683-739, 1991.

[26] T. D. Dewit, A. L. Pecquet, and J. C. Vallet, The biorthogonal decomposition as a tool for invetigation fluctuations in plasmas ., Physics of Plasmas, vol. 1, no. 10, pp. 32883300, 1994.

[27] B. F. Feeny and R. Kappagantu, On the physical interpretation of proper orthogonal modes in vibrations, Journal of Sound and Vibration, vol. 211, no. 4, pp. 607-616, 1998.

[28] B. F. Feeny, On proper orthogonal coordinates as indicators of modal activity, Journal of Sound and Vibration, vol. 255, no. 5, pp. 805-817., 2002.

[29] S. Han and B. F. Feeny, Application of proper orthogonal decomposition to structural vibration analysis, Mechanical Systems and Signal Processing, vol. 17, no. (5), pp. 9891001., 2003.

[30] G. Kerschen and J. C. Golinval, Physical interpretation of the proper orthogonal modes using the singular value decomposition, Journal of Sound and Vibration, vol. 249, no. 5, pp. 849-865., 2002.

[31] U. Iemma, L. Morino, and M. Diez, Digital holography and karhunen-loeve decomposition for the modal analysis of two-dimensional vibrating structures, Journal of Sound and Vibration, vol. 291, pp. 107-131, 2006.

[32] B. F. Feeny and Y. Liang, Interpreting proper orthogonal modes in randomly excited vibration systems, Journal of Sound and Vibration, vol. 265, no. 5, pp. 953-966., 2003.

[33] A. V. Oppenheim and R. W. Schafer, Discrete-Time Signal Processing. Prentice Hall, Englewood Cliffs, NJ., 1989. 
[34] R. Gilmore and M. Lefranc, The Topology of Chaos. Wiley, New York., 2002.

[35] I. T. Georgiou and C. I. Papadopoulos, Developing pod over the complex plane to form a data processing tool for finite element simulations of steady state structural dynamics, in International Mechanical Engineering Congress and Exposition, on DVD ROM, November 5-10 2006.

[36] L. Rayleigh, The Theory of Sound, vol. 1. New York: reprinted by Dover 1945, 1877.

[37] T. K. Caughey, Classical normal modes in damped linear systems., Journal of Applied Mechanics, vol. 27, pp. 269-271, 1960. Transactions of the ASME 82, series E.

[38] L. Meirovitch, Principles and Techniques in Vibrations. Prentice Hall., Upper Saddle River, NJ., 1997.

[39] J. Ginsberg, Mechanical and Structural Vibrations,. Wiley, New York., 2001. 